\title{
Free radical scavenging activity of methanolic and aqueous extract of Albizia lebbeck leaves
}

\author{
P. Joshi Umesh, A. Jain Swapnesh, P. Chittam Kailaspati, S. Patil Minal, D. Wagh Rajendra \\ Department of Pharmacognosy, DCS's A.R.A. College of Pharmacy, Nagaon, Dhule, Maharashtra, India
}

\begin{abstract}
Antioxidants are emerging as prophylactic and therapeutic agents for various diseases. The present study deals with an antioxidant potential of methanolic and aqueous extract of Albizia lebbeck leaves. The antioxidant potential of methanolic and aqueous extract of $A$. lebbeck leaves was investigated by employing two in vitro methods, such as Nitric oxide radical scavenging activity and reducing power assay. Results obtained in the present study reveal methanolic extract of $A$. lebbeck possesses significant antioxidant activity as compared to aqueous extract.
\end{abstract}

Correspondence:

Joshi Umesh Prabhakar, Department of

Pharmacognosy, DCS's A.R.A. College of

Pharmacy, Nagaon, Dhule, Maharashtra, India.

E-mail: joshiup2008@gmail.com
Keywords: Albizia lebbeck leaves, antioxidant, nitric oxide radical scavenging, reducing power assay etc.

\section{Introduction}

Ever since the birth of humanity, there has been a relationship between life, disease, and plants. Primitive men started studying diseases and treatments. There is no record that people in prehistoric times used synthetic medicines for their aliments but they tried to make use of the things they could easily procure. The most common thing they could find was there in environment, i.e., the plants and animals. They started using plants and found that majority of plants were suitable as food, whereas other were either poisonous or medicinally useful. By their experience, this knowledge of herbal remedies was transferred to generation as folk medicine. Hence, the history of herbal medicine is as old as human history. Herbal medicine is still the mainstay of about $75-80 \%$ of the world's population, mainly in the developing countries, for primary health care because of better cultural acceptability, better compatibility with the human body, and lesser side effects. It is estimated that approximately one-quarter of prescribed drugs contain plant extracts or active ingredients obtained from or modeled on plant substances. ${ }^{[1]}$

\section{Access this article online}

Website: http://www.jpbs-online.com E-ISSN: 2321-0125 DOI: $10.31555 / j p b s / 2018 / 6 / 1 / 9-12$

How to cite this article: Umesh PJ, Swapnesh AJ, Kailaspati PC, Minal SP, Rajendra DW. Free radical scavenging activity of methanolic and aqueous extract of Albizia lebbeck leaves. J Pharm BioSci 2018;6(1):9-12.

Source of Support: Nil, Conflict of Interest: None declared.
Higher plants have been used as a source of drugs by humanity for several 1000 years. In fact, ancient man was totally dependent on green plants for his day-to-day needs of medicaments. With the development of modern medicine, synthetic drugs and antibiotics, the importance of plants as raw material for drugs decreased considerably. However, plants were used as a basis of some of the most important drugs, even in the modern system of medicine. With the advancement of synthetic organic chemistry, most of the active constituents of plants used in medicine were synthesized. At one time, it was thought that ultimately all the plant drugs would be obtained from synthetic sources. However, in spite of phenomenal progress in the development of new drugs from synthetic sources and the appearance of antibiotics as major therapeutic agents, plants continue to provide basic raw materials for some of the most important drugs. ${ }^{[2]}$

Today in many countries, modern medicine has displaced plants with many synthetic products but almost 30\% of pharmaceutical preparations are still obtained directly or indirectly from plants. The modern era has seen some decline in the use of medicinal plants and their extracts as therapeutic agent, particularly in developed countries, many of which either been discarded by the medical profession or now given in the form of isolated compound. ${ }^{[3]}$

Antioxidant means "against oxidation" and the work to protect lipid from peroxidation by radicals. The human body as an elaborate antioxidant defense system. The main characteristics of an antioxidant are its ability to trap free radicals. Highly reactive free radicals and oxygen species are present in biological system from a wide variety of sources. These free radicals may oxidize nucleic acid, proteins, lipids, or DNA and can initiate degenerative disease. An antioxidant compound like phenolic acids, polyphenols, and flavonoid scavenge 
free radicals such as peroxide, hydroperoxide, or lipid peroxyl and thus inhibit the oxidative mechanism that leads to degenerative diseases. There are a number of clinical studies suggesting that the antioxidant in fruits, vegetables, tea, and red wine are the main factors for the observed efficacy of these foods in reducing the incidence of chronic diseases including heart disease and some cancers. ${ }^{[4]}$

Free radicals are defined as any atom or molecule having unpaired electrons. They are involved in various physiological processes (inhibition of peroxidation of the membrane lipids, inhibition of mitochondrial respiratory chain enzymes, etc.) and play a major role in the inception of many diseases and ultimately lead to cell death. They also affect the food's sensory quality-color, taste, and texture, which also shorten the shelf life and can result in rejection on the part of consumers. Free radicals are implicated for many diseases including diabetes mellitus, arthritis, cancer, and aging, in the treatment of these diseases antioxidant therapy has gained utmost importance. ${ }^{[5]}$

Many herbal remedies individually or in combination have been recommended in various medical expositions for the cure of different diseases. Plant product serves the best source for controlling these activities by its own metabolic pathway. Currently, there has been an increased interest globally to identify an antioxidant compound that is pharmacologically potent and has low or no side effects. As plants are a source of natural antioxidants, much attention has been gain to plants. A variety of free radicals scavenging antioxidants exists within the body in which many of them are derived from dietary sources such as fruits, vegetables, and teas. Dietary antioxidants are defined as a substance in foods that significantly decreases the adverse effect of reactive species, such as reactive oxygen and nitrogen species, on normal physiological function in humans. Natural antioxidants have gained increasing interest among consumers and the scientific community because they are economic, does not reduce food quality and do not exert side-effects like synthetic antioxidants. The antioxidant property may be related to the phenols and flavonoids present in the extracts. ${ }^{[6]}$

\section{Material and Methods}

\section{Collection and identification of plant material}

Albizia lebbeck leaves were collected from local area of District-Dhule, Maharashtra, India. The identity of the plant material was verified by Dr. D. A. Dhale, Head, SSVPS's Science College, Dhule, Maharashtra, India. A voucher specimen (No-1) is deposited in the herbarium of SSVPS's Science College, Dhule, Maharashtra, India. The plant material was dried in a hot air oven $\left(<50^{\circ} \mathrm{C}\right)$, stored in airtight glass bottles and powdered to 40 mesh.

\section{Extraction}

The dried powdered plant material was submerged in sufficient volume of methanol and aqueous in an air-tight flat bottomed container for 7 days, with occasional shaking and stirring. The extracts were then filtered evaporated on electrical water bath and dried in vacuum oven.

\section{Chemicals}

Sodium nitroprusside solution, sulphanilamide, O-phosphoric acid, naphthyl ethylenediamine dihydrochloride, standard phosphate buffer, ascorbic acid, potassium ferricyanide, trichloroacetic acid, ferric chloride solution $(0.1 \% \mathrm{w} / \mathrm{v})$.

\section{In vitro antioxidant evaluation}

a. Nitric oxide (NO) radical scavenging activity ${ }^{[7,8]}$

Prepare different concentrations of extract in standard phosphate buffer in concentration range 25, 50, 75, 100, 125, 150, 175, and $200 \mu \mathrm{g} / \mathrm{ml}$. Mix. $1 \mathrm{ml}$ sodium nitroprusside $(10 \mathrm{mM})$ in phosphate buffered saline with $1 \mathrm{ml}$ of each extract and standard ascorbic acid separately and incubate at room temperature for $150 \mathrm{~min}$. The same reaction mixture without the extract sample but with an equivalent amount of standard phosphate buffer should be serving as control. After the incubation period, to $1.5 \mathrm{ml}$ of above reaction mixture; add $0.5 \mathrm{ml}$ of Griess reagent. Immediately measure the absorbance of the chromophore formed at $546 \mathrm{~nm}$. Determine the percentage scavenging activity at different concentrations. Carry out the same assay in triplicate.

b. Reducing power assay. ${ }^{[9,10]}$

The reducing power of methanolic and aqueous extract was determined. Different concentration of methanolic and aqueous extract $(25,50,75, \ldots .200 \mu \mathrm{l} / \mathrm{ml})$ was prepared and mix $1.0 \mathrm{ml}$ of each sample with $2.5 \mathrm{ml}$ of phosphate buffer solution (50 mM, pH 7.0) and $2.5 \mathrm{ml}$ of $1 \%$ potassium ferric cyanide separately, incubate at $50^{\circ} \mathrm{C}$ for $20 \mathrm{~min}$. Then add $2.5 \mathrm{ml}$ of trichloroacetic acid (10\%) to the mixture, centrifuge at $3000 \mathrm{rpm}$ for $10 \mathrm{~min}$. Finally, mix $1.25 \mathrm{ml}$ from supernatant with $1.25 \mathrm{ml}$ of distilled water and $0.25 \mathrm{ml}$ of $\mathrm{FeCl}_{3}$ solution $(0.1 \% \mathrm{w} / \mathrm{v})$. Immediately measure the absorbance at $700 \mathrm{~nm}$. Carry out the same assay in triplicate. Prepare the different concentration of sample results of this method is based on the fact of increased absorbance value of different concentrations indicates higher reducing power.

\section{Statistical analysis}

The data presented as Mean \pm SEM. The activities of all extracts were compared with the control. All the extracts showed significant activity with a higher duration of paralysis and death. Values of $P<0.001$ were considered statistically significant.

\section{Result and Discussion}

In Nitric oxide radical scavenging activity model, it is observed that methanolic and aqueous extract of leaves of $A$. lebbeck have demonstrated dose dependent increase in the nitric oxide anion scavenging property. The methanolic extract shows potential antioxidant activity as compared to aqueous extract shows $88.17 \pm 0.62$ and $79.19 \pm 0.62$ percentage inhibition at $200 \mu \mathrm{g} / \mathrm{ml}$ respectively and ascorbic acid has shown $95.26 \pm 0.24$ percentage inhibition at $200 \mu \mathrm{g} / \mathrm{ml}$ as shown in Table 1 . The IC50 value for methanolic and aqueous extract and standard ascorbic acid as shown in Table 2. 
Table 1: Nitric oxide radical scavenging activity of methanolic and aqueous extract of plant Albizia lebbeck with standard ascorbic acid (percentage inhibition)

\begin{tabular}{lccc}
$\begin{array}{l}\text { Concentration } \\
(\mu \mathrm{g} / \mathrm{ml})\end{array}$ & $\begin{array}{c}\text { Methanolic extract } \\
(\% \text { inhibition) }\end{array}$ & $\begin{array}{c}\text { Aqueous extract } \\
(\% \text { inhibition) }\end{array}$ & $\begin{array}{c}\text { Ascorbic acid } \\
(\% \text { inhibition })\end{array}$ \\
\hline 25 & $29.54 \pm 0.62 *$ & $23.16 \pm 0.62 *$ & $38.76 \pm 0.85 *$ \\
50 & $39.94 \pm 0.47 *$ & $30.49 \pm 1.08 *$ & $52.24 \pm 2.25 *$ \\
75 & $48.69 \pm 0.62 *$ & $40.18 \pm 0.23 *$ & $59.09 \pm 0.62 *$ \\
100 & $58.38 \pm 0.23 *$ & $49.16 \pm 0.85 *$ & $64.76 \pm 0.62 *$ \\
125 & $65.71 \pm 0.62 *$ & $57.67 \pm 0.85 *$ & $71.17 \pm 0.63 *$ \\
150 & $73.27 \pm 0.23 *$ & $65.47 \pm 0.85 *$ & $81.79 \pm 0.62 *$ \\
175 & $79.90 \pm 0.62 *$ & $72.57 \pm 1.24 *$ & $89.59 \pm 0.63 *$ \\
200 & $88.17 \pm 0.62 *$ & $79.19 \pm 0.62 *$ & $95.26 \pm 0.24 *$ \\
\hline
\end{tabular}

*Data and results are expressed as mean \pm SEM and mean is representation of three experiments, Albizia lebbeck:A. lebbeck

Table 2: $\mathrm{IC}_{50}$ result nitric oxide radical scavenging activity of various extracts of leaves of Albizia lebbeck with standard ascorbic acid

\begin{tabular}{lc} 
Extract & IC $_{50}$ \\
Methanolic & $101.21 \mu \mathrm{g} / \mathrm{ml}$ \\
Aqueous & $114.68 \mu \mathrm{g} / \mathrm{ml}$ \\
Ascorbic acid & $90.58 \mu \mathrm{g} / \mathrm{ml}$ \\
\hline
\end{tabular}

Calculated by regression equation, Albizia lebbeck: A. lebbeck

Table 3: Observation of reducing power determination of ascorbic acid, methanolic, and aqueous extract of leaves of Albizia lebbeck

\begin{tabular}{llcc}
$\begin{array}{l}\text { Concentration } \\
(\boldsymbol{\mu g} / \mathbf{m l})\end{array}$ & \multicolumn{3}{c}{ Absorbance $(\mathbf{n m})$} \\
\cline { 2 - 4 } & Ascorbic acid & $\begin{array}{c}\text { Methanolic } \\
\text { extract }\end{array}$ & $\begin{array}{c}\text { Aqueous } \\
\text { extract }\end{array}$ \\
\hline 5 & $0.035 \pm 0.0008 *$ & $0.029 \pm 0.0847 *$ & $0.028 \pm 0.0010 *$ \\
50 & $0.051 \pm 0.0005 *$ & $0.047 \pm 0.0008 *$ & $0.037 \pm 0.0011 *$ \\
75 & $0.069 \pm 0.0005 *$ & $0.058 \pm 0.0006 *$ & $0.043 \pm 0.0003 *$ \\
100 & $0.077 \pm 0.0008 *$ & $0.071 \pm 0.0003 *$ & $0.052 \pm 0.0003 *$ \\
125 & $0.090 \pm 0.0006 *$ & $0.075 \pm 0.0003 *$ & $0.064 \pm 0.0008 *$ \\
150 & $0.099 \pm 0.0008 *$ & $0.092 \pm 0.0006 *$ & $0.078 \pm 0.0003 *$ \\
175 & $0.136 \pm 0.0008 *$ & $0.096 \pm 0.0003 *$ & $0.082 \pm 0.0008 *$ \\
200 & $0.171 \pm 0.0011 *$ & $0.108 \pm 0.0008 *$ & $0.091 \pm 0.0011 *$ \\
\hline $\begin{array}{l}\text { *Data and results are expressed as mean } \pm \text { SEM and mean is representation of three experiments, } \\
\text { Albizia lebbeck: } A \text {. lebbeck }\end{array}$ & & &
\end{tabular}

It is observed that the both methanolic and aqueous extract of plant A. lebbeck demonstrated dose dependant increase in the reducing property. To find the active species which is capable of donating hydrogen and subsequently its leads to the reducing power activity was determine. The high reducing power is indicative of the hydrogen donating ability of the active species present in extract. The reducing power assay of various extract of plant $A$. lebbeck was estimated by using potassium ferricyanide reduction method. In the present study the reducing power of the methanolic extract of plant $A$. lebbeck was found to be excellent and steadily increase in direct proportional to the increasing concentration extract as compare to other extract in comparison with standard ascorbic acid. The reducing power of methanolic, aqueous and standard ascorbic acid at concentration $200 \mu \mathrm{g} / \mathrm{ml}$ was found to be $0.108,0.091$ and 0.171 respectively as shown in Table 3.
Nitric oxide is an important chemical mediator generated by endothelial cells macrophages, neurons and is involved in regulation of various physiological processes. Excess concentration of nitric oxide is associated with several diseases. Nitric oxide is generated in biological tissues by specific nitric oxide synthesis (NOS) which metabolized arginine to adrenaline with formation of nitric oxide via a five electron oxidative reaction. These compounds are responsible for altering the structural and functional behavior of many ocular components.

The percentage scavenging activity increased with increasing concentration of the extract. Lower the $\mathrm{IC}_{50}$ value indicates better is the scavenging ability of the sample. The nitric oxide scavenging activity of methanol extract showed better activity than other extract, however standard ascorbic acid activity was significantly higher than that of all extract. The reducing power of extract of A. lebbeck was found remarkable and the reducing power of the extract was observed to rise as the concentration of the extract gradually increased.

The reducing power is associated with antioxidant activity and many serve as a significant reflection of the antioxidant activity. Compound with reducing power indicate that they are electron donors and can reduce the oxidized intermediates of lipid preoxidation processes, so that they can act as primary and secondary antioxidants. In this assay the yellow color of test solution changes to various shades of green and blue depending on the reducing power of each compound, presence of reducers couses the conversion of $\mathrm{Fe}^{+3} /$ ferricyanide complex used in this method to ferrous form. The high reducing power is indicative of the hydrogen donating ability of the active species present in the extract.

Reducing power of the fractions was assessed using ferric to ferrous reducing activity as determined spectrophotometrically from formation of blue color complex. Reducing power of different extract of plant $A$. lebbeck was compared with standard ascorbic acid. Methanolic extract exhibited most reducing power. This extract indicates that extract may consist of polyphenolic compounds that usually shows great reducing power.

\section{Conclusion}

We conclude from the above discussion that those methanolic extracts have antioxidant activity by scavenging the nitric oxide free radical. It is very much helpful for investigation of new drugs for various free radical generation diseases by identifying the compound isolation process.

\section{References}

1. Jayasundar R. Ayurved: A distinctive approach to health. Curr Sci 2010;98:909.

2. Mathew J, Arora KM, Muzumdar A, Kumar G, Karthik L, Raw KV. Evaluation of phytochemical composition and antioxidant activity of aqueous extract of Barleria mysorensis and Furcraea foetida leaves. Res J Pharm Tech 2012;5:1503-4.

3. Vyas BA. Phytopharmacological Action of Pergularia daemia with Special Reference to its Actions and Mechanism of Action as Diuretic and Antiinflammatory Agent. PhD Thesis Submitted to Veer Narmad South Gujarat 
Umesh, et al.: Free radical scavenging activity

University, 5; 2010. p. 1-2.

4. Kanagavalli M, Anuradha R. A study on phytochemical constituents and in vitro antioxidant activity of Carica papaya. Res J Pharm Tech 2012;5:119-20.

5. Jain N, Goyal S, Ramawat KG. Radical scavenging activity and total phenolic content in selected fruits and vegetables. Res J Pharm Tech 2012;5:121.

6. Behera S, Babu MS, Ramani RY, Chaudhury PK. A comparative study on phytochemical investigation and antioxidant activity of polyherbal mixture of Ocimum canum and Pongamia pinnata hydro-alcoholic leaf extract. Res J Pharmacogn Phytochem 2012;4:250-61.
7. Khadbadi SS, Deore SL, Baviskar BA. Experimental Phytopharmacology - A Comprehensive Guide. Vol. 2. Pune: Nirali Prakashan; 2013. p. 27-4.29.

8. Huda N, Masum M, Hamid K, Urmi KF. In vitro antioxidant activities of different parts of the plant Moringa oleifera Lam. Res J Pharm Tech 2012;5:1532-3.

9. Shivaprasad HN, Mohan S, Kharya MD, Lakshman K. In-vitro models for antioxidant activity evaluation: A review. Pharmacogn J 2005;3:1-6.

10. Ajay S, Bhardwaj S, Mann AS, Kharya MD. Screening methods of antioxidant activity: An overview. Pharmacogn Rev 2007;1:232-8. 